\title{
Single-Center Review of Appropriateness and Utilization of Upper Endoscopy in Dyspepsia in the United States
}

\author{
Kamesh Gupta ${ }^{\text {a, d, e }}$, Kevin Groudan ${ }^{\text {a, d }}$, Kathryn Jobbins ${ }^{\text {a }}$, Bandhul Hans ${ }^{\mathrm{b}}$, Rohit Singhania ${ }^{\mathrm{c}}$
}

\begin{abstract}
Background: We compared real-world practice of dyspepsia management to the new American College of Gastroenterology (ACG)/ Canadian Association of Gastroenterology (CAG) guidelines 2017.

Methods: We conducted a retrospective, observational study using administrative data to include patients undergoing esophagogastroduodenoscopy (EGD) for dyspepsia.

Results: Out of 122 EGDs, only 30 (24.5\%) were deemed appropriate per guidelines. Only 13 (14.1\%) patients had undergone both Helicobacter pylori (H. pylori) test and treat and adequate proton pump inhibitor (PPI) before undergoing endoscopy. Nineteen (15.5\%) patients had alarm symptoms (weight loss, melena and early satiety). Positivity rate of $H$. pylori was $36.3 \%$, but only half completed treatment. Twenty-six patients $(21.3 \%)$ had abnormalities on endoscopy, most commonly gastritis. There were no cases of gastric/esophageal cancer.
\end{abstract}

Conclusions: The rate of inappropriate upper gastrointestinal endoscopy is higher than the existing literature, likely because of the stricter use of EGD in patients $<60$ years. Only one in eight patients underwent the recommended workup before undergoing endoscopy.

Keywords: Dyspepsia; Guidelines; Value-based care; Upper endoscopy; United States

\section{Introduction}

Dyspepsia encompasses a constellation of upper abdominal

Manuscript submitted February 9, 2021, accepted March 27, 2021

Published online April 21, 2021

aDepartment of Medicine, University of Massachusetts-Baystate, Springfield, MA, USA

bepartment of Medicine, Allegheny General Hospital, Boston, MA, USA 'Division of Gastroenterology, Department of Medicine, University of Massachusetts-Baystate, Springfield, MA, USA

${ }^{\mathrm{d}}$ These authors contributed equally to this manuscript.

${ }^{e}$ Corresponding Author: Kamesh Gupta, Department of Internal Medicine, UMMS-Baystate Medical Center, 759 Chestnut St, Springfield, MA 01199,

USA. Email: Kameshg9@gmail.com

doi: https://doi.org/10.14740/gr1370 symptoms, with a pooled prevalence of $21 \%$ [1]. The symptoms of dyspepsia can be confused with other digestive disorders such as peptic ulcer disease (PUD), gastroesophageal reflux disease (GERD), motility disorders and other functional disorders such as epigastric pain syndrome, postprandial syndrome and irritable bowel syndrome. A focused history ruling out dietary, medical and medication-related causes as well as endoscopic evaluation to exclude structural disease is necessary to establish a diagnosis. One of the concerning mimickers of epigastric pain is gastric malignancy, for which an upper endoscopy is usually performed [2]. Dyspepsia is the most common indication for esophagogastroduodenoscopy (EGD), accounting for $50 \%$ of all such procedures [3-5]. However, in the majority of cases, the endoscopic evaluation reveals no underlying organic lesion, also termed as functional dyspepsia [6-8]. Dyspepsia is estimated to cost the US health care service over $\$ 18$ billion per annum [9].

American Society of Gastrointestinal Endoscopy (ASGE) published guidelines in 2015 on the role of endoscopy in patients with dyspepsia, whereby patients aged more than 50 years and/or patients with alarm symptoms were appropriate to undergo EGD [10]. More recently, American College of Gastroenterology (ACG) and the Canadian Association of Gastroenterology (CAG) published joint guidelines in 2017 for the management of dyspepsia that clearly laid down noninterventional, effective and affordable steps to undertake before undergoing endoscopy, as the endoscopy was found to be of low yield in true dyspepsia patients [11]. It is estimated that up to $56 \%$ of diagnostic upper gastrointestinal endoscopy procedures are inappropriate $[12,13]$ and do not follow with current guidelines. The large burden of inappropriate referrals for endoscopy for dyspepsia leads to both unacceptable consumption of health resources and detrimental increase of waiting time for clinically indicated patients. In this study, we report on the appropriateness of endoscopy for dyspepsia, medical management undertaken before undergoing EGD and the prevalence of medical and psychiatric comorbidities in patients with dyspepsia.

\section{Materials and Methods}

This retrospective study was conducted at an independent, integrated academic health system in western Massachusetts, serving a diverse population. For the purpose of the study, we used administrative data to identify 250 patients aged $\geq$ 18 years who were referred for EGD from three health-system 
associated clinics. All patients underwent EGD from January 2018 to December 2018. Patients were included if the primary indication for undergoing the EGD was dyspepsia, as defined by Rome III criteria [14]. Rome III criteria define dyspepsia as one or more of the following three symptoms for 3 months within the first 6 months of symptom onset: postprandial fullness, early satiety and epigastric pain or burning. We excluded patients who had a pathologic diagnosis made on a previous endoscopy. Since the symptoms often overlap with those of PUD, we individually reviewed each chart to exclude patients whose primary complaint was: 1) Retrosternal burning pain, which would suggest a more appropriate diagnosis of gastroesophageal reflux disease (as per GERD questionnaire); 2) Progressive dysphagia and/or weight loss in the absence of epigastric pain; and 3) Jaundice or history of pancreatic cancer. After exclusions, the study population was 122 patients. This study was reviewed and approved by the IRB of UMMS-Baystate. This paper was exempt from ethical committee approval as it does not contain any studies involving human participants or animals.

For the remaining 122 patients, their charts were reviewed and the following information was extracted: 1) Baseline characteristics, including medical and psychiatric comorbidities; 2) Helicobacter pylori (H. pylori) testing performed, and if positive, were patients treated appropriately; 3 ) proton pump inhibitor (PPI) used, and for what duration; 4) Other therapies tried such as promotility drugs and tricyclic antidepressants (TCAs); and 5) Referral for EGD by primary care MD/DO or NP/PA. Based on the documented management steps taken before undergoing the EGD, the appropriateness of the test was assessed using the $\mathrm{ACG} / \mathrm{CAG}$ guidelines for dyspepsia [11]. To mitigate any potential for bias, charts were retrospectively reviewed by two authors (KG1 and KG2) to assess patients meeting the diagnostic criteria for dyspepsia, in order to minimize the selection bias. Decisions regarding appropriateness were subsequently made in the original clinical chart. The appropriateness of EGDs was classified into two categories: likely appropriate and probable overuse. We further examined findings from the EGD and compared these between the appropriate and the overuse groups.

\section{Statistical analysis}

Differences in key outcomes were assessed using the Fisher's exact test, as several individual values were less than 5 , and considered statistically significant at a $\mathrm{P}$ value $<0.05$. All statistical analyses were performed using STATA 15.0 (SAS Institute, Inc., Cary, NC).

\section{Results}

A total of 122 EGDs were performed for dyspepsia and 30 $(24.5 \%)$ were performed in patients $>60$ years of age. The mean age of the study population was 47.6 years, and $65.5 \%$ (n $=80$ ) were female. Table 1 shows the baseline characteristics and comorbidities. We found that patients who underwent an
EGD not indicated per guidelines included a higher proportion of younger patients $<40$ years $(34 \%$ vs. $0 \%, \mathrm{P}<0.001)$ and a longer duration of symptoms ( $>1$ year, $37 \%$ vs. $16.6 \%, \mathrm{P}=$ $0.044)$. There was no difference found between the two groups on the basis of referring providers.

According to the ACG/CAG guidelines, in the cases we reviewed, endoscopy was not considered to be indicated in 92 cases $(75.4 \%)$. These included all the patients aged $<60$ years as according to these guidelines. In the 92 patients aged $<60$ years, only $13(14.1 \%)$ patients had undergone the appropriate additional treatments, i.e., both $H$. pylori test and treat and PPI for at least a month before undergoing endoscopy. Of the patients $<60$ years of age, $20.6 \%(n=19)$ had alarm symptoms. These were weight loss $(14.1 \%, \mathrm{n}=13)$, melena $(3.3 \%, \mathrm{n}=3)$ and early satiety $(3.3 \%, \mathrm{n}=3)$. Three $(3.3 \%)$ had documented family history of gastric cancer and none had any information on belonging to a high risk region.

In the entire cohort of 122 patients, 33 patients (27\%) had H. pylori testing, of which $12(9.8 \%)$ were positive. Howev$\mathrm{er}$, of these 12 patients, only half of them $(\mathrm{n}=6)$ completed treatment before undergoing endoscopy. Seventy-two patients $(59 \%)$ were trialed on PPI, of whom 24 had treatment for less than 1 month, 30 took it for $1-6$ months and 20 took it for > 6 months. Other treatments included $\mathrm{H}_{2}$-blockers and carafate in two patients each. Tricyclic antidepressants were tried in only one patient and no cases had any promotility drug use recorded. These findings are summarized in Table 2.

In 82 patients (67.2\%), endoscopy did not show any abnormalities. Forty patients had positive findings on endoscopy. Most common of these were: 1) Esophagitis/gastritis/duodenitis in 28 patients $(23 \%)$; 2) Hiatal hernia in four patients $(3.3 \%)$; 3 ) Peptic ulcer disease was only noted in two patients $(1.6 \%)$; and 4) Barrett's esophagus was noted in two patients (2.6\%). Eleven patients tested positive on endoscopic biopsies for $H$. pylori. No complications were reported because of the procedure.

In Table 3, we report the endoscopic diagnoses of both appropriate and inappropriate endoscopies. The appropriate upper gastrointestinal endoscopies yielded $63.3 \%$ of relevant findings, whereas inappropriate endoscopies yielded $22.8 \%$ relevant lesions. The difference was statistically significant ( $\mathrm{P}$ $<0.001)$. Significantly more hiatal hernia was found in the "appropriate upper gastrointestinal endoscopy" group. A higher percentage of esophagitis/gastritis/duodenitis, celiac disease, Schatzki ring, peptic ulcer and Barrett's esophagus was found in the "appropriate upper gastrointestinal endoscopy" group, but the difference was not statistically significant. No gastric cancer was found in our cohort.

\section{Discussion}

This retrospective observational study found that overuse of upper gastrointestinal endoscopy was common at our independent, integrated academic health system in western Massachusetts, occurring in about $75 \%$ of patients with dyspepsia, according to the latest guidelines provided by ACG/CAG. Guidelines on the appropriateness of endoscopic procedures are updated regularly with the latest available data to guide 
Table 1. Characteristics of Patients Undergoing EGD for Dyspepsia, $n$ (\%)

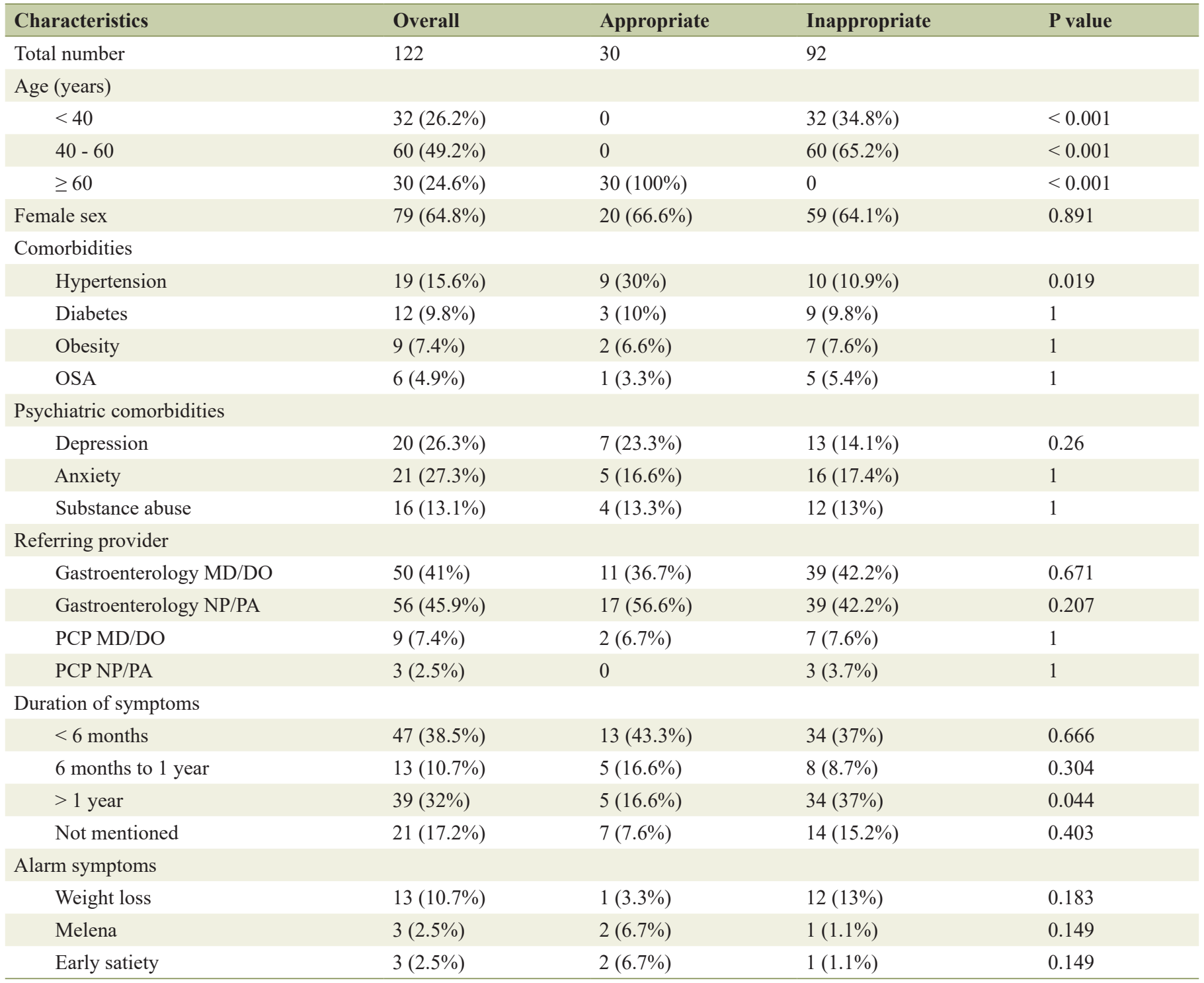

EGD: esophagogastroduodenoscopy; OSA: obstructive sleep apnea.

clinicians in judicious choices for patients $[10,11]$. Several studies show a substantial rate of inappropriateness for the indication of upper endoscopy, widely ranging from $5 \%$ to $62 \%$ [12-18]. The only known study looking at the appropriateness of upper endoscopy for dyspepsia patients only was performed in 2002 by Manes et al [19]. This study was performed in southern Italy and found the rate of inappropriate endoscopies to be $50 \%$ according to Maastricht Consensus.

We found that the rate of EGDs in patients with dyspepsia, not indicated as per ACG/CAG guidelines, was $75 \%$ in a Western Massachusetts health system. The higher rate of inappropriate studies in our study is likely because of the stricter use of EGD in patients $<60$ years wherein ACG/CAG guidelines recommend that endoscopy be of high yield in this patient group if they have a family history of gastric cancer or spent their childhood in a high risk region, mainly East Asia. These guide- lines are different from the previous ACG guidelines in 2005, in that the threshold for performing EGD was raised from 55 to 60 years [20]. Furthermore, now EGD is not suggested for patients with dyspepsia under the age of 60 even if they are exhibiting alarm symptoms. However, both of these are conditional recommendations, made due to the low yield of upper endoscopy in patients $<60$ years even with alarm symptoms, based upon a meta-analysis [21]. Our study shows that there is a need for improving guideline-based care in managing for patients with dyspepsia. Various strategies have been recommended to facilitate implementation of practice guidelines, including the use of performance measures based on practice guidelines, chart audits with feedback of results, reminder systems (including pathways and tools) and educational outreach visits [22].

As our study shows, physicians still rely on upper gastrointestinal endoscopy to assign a suitable treatment for pa- 
Table 2. Real-World Management of Patients With Dyspepsia

\begin{tabular}{|c|c|}
\hline Intervention & Total number (\%) \\
\hline \multicolumn{2}{|l|}{ H. pylori test } \\
\hline Positive & $12(9.8 \%)$ \\
\hline Treatment completed & $6(4.9 \%)$ \\
\hline Negative & $18(14.8 \%)$ \\
\hline Not performed & $92(75.4 \%)$ \\
\hline \multicolumn{2}{|l|}{ Other diagnostic tests performed } \\
\hline Celiac testing & $3(2.5 \%)$ \\
\hline Gastric emptying study & $2(1.6 \%)$ \\
\hline Right upper quadrant US/HIDA & $2(1.6 \%)$ \\
\hline SIBO testing & $1(0.8 \%)$ \\
\hline \multicolumn{2}{|l|}{ PPI used } \\
\hline$<6$ months & $23(18.6 \%)$ \\
\hline 6 months to 1 year & $23(18.6 \%)$ \\
\hline$>1$ year & $17(13.9 \%)$ \\
\hline Not used & $47(38.5 \%)$ \\
\hline \multicolumn{2}{|l|}{ Other treatments } \\
\hline $\mathrm{H}_{2}$-blockers & $6(4.8 \%)$ \\
\hline Mag sulfate & $3(2.4 \%)$ \\
\hline Sucralfate & $2(1.6 \%)$ \\
\hline Amitriptyline & $1(0.8 \%)$ \\
\hline Miralax & $1(0.8 \%)$ \\
\hline Previous endoscopy performed & $7(5.7 \%)$ \\
\hline
\end{tabular}

US: ultrasonography; HIDA: hepatobiliary iminodiacetic acid; SIBO: small intestinal bacterial overgrowth; PPI: proton pump inhibitor.

tients with dyspepsia. A survey of gastroenterologists looked into the reasons for overuse of surveillance endoscopies, and found medico legal concerns as a chief reason for this practice, in addition to procedure reimbursement and patient preference [22]. In our population cohort, almost a quarter patients were aged less than 40 years, and almost two-thirds were females. We showed that there was no difference in guideline-guided therapy among patients referred for endoscopy by primary care physicians, or gastroenterologists. This finding is different from previous literature, where gastroenterologists had a better rate of following guidelines [10]. The change could be because ACG/CAG has further simplified the criteria for undergoing EGD. Furthermore, there was no difference between physicians and advanced practitioners as well. Our study again showed that the presence of alarm symptoms did not correlate with significant endoscopic findings. This event has previously been shown to be true in multiple studies, thus leading to the latest change in the ACG/CAG guidelines [8, 23].

Our study confirmed again that the rates of testing of $H$. pylori are abysmally low, performed in only a quarter of the total patients in our cohort. The low rates of outpatient testing for $H$. pylori could be explained as the preferred mode of $H$. pylori testing in gastric biopsy, accounting for $59 \%$ of total $H$. pylori tests in a recent survey [24]. This reflects the practice of referral of patients with dyspepsia before undergoing outpatient testing for $H$. pylori by non-invasive methods such as urea breath testing (UBT) and stool antigen testing for active infection, and serology for active or past infections. H. pylori infection has been demonstrated to have higher prevalence in patients with dyspepsia, possibly leading to inflammation of the gastric mucosa, and chronic gastritis [25, 26]. However, in clinical practice, the results of $H$. pylori eradication have varied. Randomized controlled trials of $H$. pylori eradication therapy versus placebo report that only a proportion (10-12\%) of functional dyspeptic patients achieve a significant improvement of persistent symptoms after $H$. pylori eradication [27]. Furthermore, these results may take up to a year to manifest. However, De Jong et al summarized six studies that showed a considerable proportion (68-94\%) did not need additional upper gastrointestinal endoscopy after either $H$. pylori eradication or, if uninfected, empirical treatment with PPIs, $\mathrm{H}_{2}-$ antagonists, prokinetics or no treatment [28]. Ten percent of our cohort tested positive for $H$. pylori before undergoing endoscopy, with a total positivity rate of $36 \%$. Only half of the patients completed the eradication therapy for $H$. pylori before undergoing EGD. A prospective study from Italy found that standardizing the implementation of guidelines amongst general practitioners for the care of patients with dyspepsia led to decrease in acid-suppression therapy, but a small increase in

Table 3. Finding on EGD and Comparison Based Upon the Appropriateness of the EGD

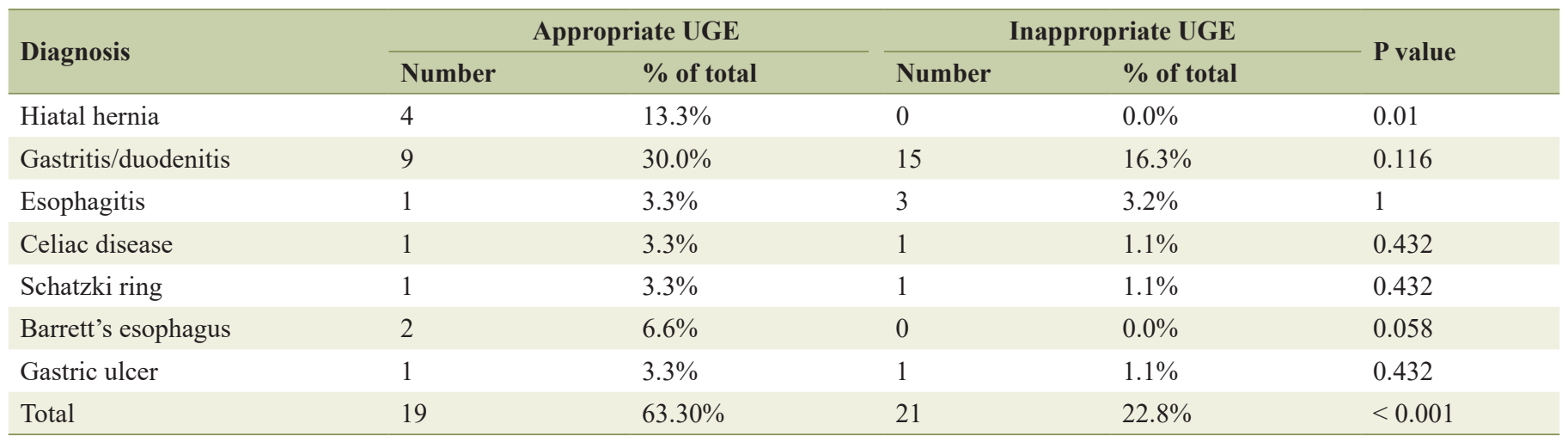

EGD: esophagogastroduodenoscopy; UGE: upper gastrointestinal endoscopy. 
referrals and EGDs [29].

Prescription rates for PPI fared slightly better, with onethird patients taking it for at least 6 months before undergoing EGD. Our study shows that the adoption of newer therapies for dyspepsia, such as TCAs, as recommended by ACG/CAG guidelines was low, tried in only one patient. A systematic review identified three trials that showed a significant effect in reducing dyspepsia symptoms (risk ratio: 0.74 ; $95 \%$ confidence interval (CI): 0.61 - 0.91) [23]. Our study also showed that almost half the population with dyspepsia had an associated psychiatric disorder. This might be why the addition of TCAs might have potential to improve symptoms and would be areas for future study. Current guidelines recommend a trial of PPI, followed by TCAs, and further by promotility drugs.

Abnormal endoscopic findings with important lesions were observed in only a small proportion of our study population, which is similar to previous reports $[10,12]$. Gastric cancer was not detected in any cases. Total numbers of abnormal endoscopic findings were significantly higher in the appropriate group than the inappropriate group per ACG/CAG guidelines. None of the differences in individual findings tended to significance, but Barrett's esophagus was detected only in the appropriate group.

The limitations of the present study included a relatively small sample size and the small number of important endoscopic lesions that were found, resulting in a low power to detect any clinically significant differences. Secondly, the study was conducted in a single center which limits the external validity of the study. Third, we performed a retrospective analysis which may have imparted selection bias. To encounter this, we had charts reviewed by two reviewers and only included patients who had a consensus reached between the reviewers. Despite these limitations, ours is the first study to present realworld data on the compliance with the ACG/CAG guidelines for management of dyspepsia.

Our study adds to the previous data that significant abnormal findings in patients aged $<60$ years were few and far in between. H. pylori was frequently detected during endoscopy. This knowledge can be utilized to improve clinical practice by testing for patients using non-invasive tests to detect $H$. pylori being the cause of the patient's symptoms. Our study also showed that a significant proportion of patients underwent endoscopy before the conclusion of the recommended monthlong treatment with PPI.

In conclusion, almost three quarters of endoscopies performed were not guideline-based. Further, adherence to the guidelines was lacking in testing for $\mathrm{H}$. pylori, which was fairly prevalent in our population. Endoscopies performed according to the guidelines were significantly more likely to show abnormal endoscopy findings. We therefore, advise practitioners to adopt these guidelines when evaluating patients with dyspepsia. Such practice would avoid unnecessary procedures, improve access to care and will result in an efficient utilization of resources.

\section{Acknowledgments}

None to declare.

\section{Financial Disclosure}

None to declare.

\section{Conflict of Interest}

None to declare.

\section{Informed Consent}

Not applicable.

\section{Author Contributions}

Dr. Gupta devised the statistical analysis plan and contributed in writing the manuscript. Dr. Groudan contributed to the statistical analysis and contributed in writing the manuscript. Dr. Hans authored the discussion section of the manuscript. Dr. Jobbins performed background check and critically appraised the manuscript. Dr. Singhania was our faculty mentor who revised and edited the final manuscript. All authors provided critical feedback and helped shape the research analysis and manuscript.

\section{Data Availability}

Any inquiries regarding supporting data availability of this study should be directed to the corresponding author.

\section{References}

1. Ford AC, Marwaha A, Lim A, Moayyedi P. What is the prevalence of clinically significant endoscopic findings in subjects with dyspepsia? Systematic review and metaanalysis. Clin Gastroenterol Hepatol. 2010;8(10):830837, e831-832.

2. Mounsey A, Barzin A, Rietz A. Functional dyspepsia: evaluation and management. Am Fam Physician. 2020; 101(2):84-88.

3. Taye M, Kassa E, Mengesha B, Gemechu T, Tsega E. Upper gastrointestinal endoscopy: a review of 10,000 cases. Ethiop Med J. 2004;42(2):97-107.

4. Olokoba AB, Olokoba LB, Jimoh AA, Salawu FK, Danburam A, Ehalaiye BF. Upper gastrointestinal tract endoscopy indications in northern Nigeria. J Coll Physicians Surg Pak. 2009;19(5):327-328.

5. Olokoba $\mathrm{AB}$, Bojuwoye BJ. Indications for oesophagogastroduodenoscopy in Ilorin, Nigeria - a 30 month review. Niger J Clin Pract. 2010;13(3):260-263.

6. Lieberman D, Fennerty MB, Morris CD, Holub J, Eisen G, Sonnenberg A. Endoscopic evaluation of patients with dyspepsia: results from the national endoscopic data repository. Gastroenterology. 2004;127(4):1067-1075. 
7. Thomson AB, Barkun AN, Armstrong D, Chiba N, White RJ, Daniels S, Escobedo S, et al. The prevalence of clinically significant endoscopic findings in primary care patients with uninvestigated dyspepsia: the Canadian Adult Dyspepsia Empiric Treatment - Prompt Endoscopy (CADET-PE) study. Aliment Pharmacol Ther. 2003;17(12):1481-1491.

8. Vakil N, Moayyedi P, Fennerty MB, Talley NJ. Limited value of alarm features in the diagnosis of upper gastrointestinal malignancy: systematic review and meta-analysis. Gastroenterology. 2006;131(2):390-401; quiz 659-360.

9. Lacy BE, Weiser KT, Kennedy AT, Crowell MD, Talley NJ. Functional dyspepsia: the economic impact to patients. Aliment Pharmacol Ther. 2013;38(2):170-177.

10. Azzam NA, Almadi MA, Alamar HH, Almalki LA, Alrashedi RN, Alghamdi RS, Al-hamoudi W. Performance of American Society for Gastrointestinal Endoscopy guidelines for dyspepsia in Saudi population: prospective observational study. World J Gastroenterol. 2015;21(2):637-643.

11. Moayyedi P, Lacy BE, Andrews CN, Enns RA, Howden CW, Vakil N. ACG and CAG Clinical Guideline: Management of Dyspepsia. Am J Gastroenterol. 2017;112(7):988-1013.

12. Froehlich F, Burnand B, Pache I, Vader JP, Fried M, Schneider C, Kosecoff J, et al. Overuse of upper gastrointestinal endoscopy in a country with open-access endoscopy: a prospective study in primary care. Gastrointest Endosc. 1997;45(1):13-19.

13. Gonvers JJ, Burnand B, Froehlich F, Pache I, Thorens J, Fried M, Kosecoff J, et al. Appropriateness and diagnostic yield of upper gastrointestinal endoscopy in an openaccess endoscopy unit. Endoscopy. 1996;28(8):661-666.

14. Chan YM, Goh KL. Appropriateness and diagnostic yield of EGD: a prospective study in a large Asian hospital. Gastrointest Endosc. 2004;59(4):517-524.

15. Mahajan RJ, Barthel JS, Marshall JB. Appropriateness of referrals for open-access endoscopy. How do physicians in different medical specialties do? Arch Intern Med. 1996;156(18):2065-2069.

16. Di Giulio E, Hassan C, Marmo R, Zullo A, Annibale B. Appropriateness of the indication for upper endoscopy: a meta-analysis. Dig Liver Dis. 2010;42(2):122-126.

17. Zuccaro G, Jr., Provencher K. Does an open access system properly utilize endoscopic resources? Gastrointest Endosc. 1997;46(1):15-20.

18. Kahn KL, Kosecoff J, Chassin MR, Solomon DH, Brook $\mathrm{RH}$. The use and misuse of upper gastrointestinal endoscopy. Ann Intern Med. 1988;109(8):664-670.
19. Manes G, Balzano A, Marone P, Lioniello M, Mosca S. Appropriateness and diagnostic yield of upper gastrointestinal endoscopy in an open-access endoscopy system: a prospective observational study based on the Maastricht guidelines. Aliment Pharmacol Ther. 2002;16(1):105110.

20. Talley NJ, Vakil N, Practice Parameters Committee of the American College of G. Guidelines for the management of dyspepsia. Am J Gastroenterol. 2005;100(10):23242337.

21. O'Sullivan JW, Albasri A, Nicholson BD, Perera R, Aronson JK, Roberts N, Heneghan C. Overtesting and undertesting in primary care: a systematic review and metaanalysis. BMJ Open. 2018;8(2):e018557.

22. Hunt SA, Abraham WT, Chin MH, Feldman AM, Francis GS, Ganiats TG, Jessup M, et al. ACC/AHA 2005 guideline update for the diagnosis and management of chronic heart failure in the adult: a report of the American College of Cardiology/American Heart Association Task Force on practice guidelines (Writing Committee to update the 2001 guidelines for the evaluation and management of heart failure): developed in collaboration with the American College of Chest Physicians and the International Society for Heart and Lung Transplantation: endorsed by the Heart Rhythm Society. Circulation. 2005;112(12):e154235.

23. Lin OS, Mannava S, Hwang KL, Triadafilopoulos G. Reasons for current practices in managing Barrett's esophagus. Dis Esophagus. 2002;15(1):39-45.

24. Murakami TT, Scranton RA, Brown HE, Harris RB, Chen Z, Musuku S, Oren E. Management of helicobacter pylori in the United States: results from a national survey of gastroenterology physicians. Prev Med. 2017;100:216-222.

25. Khedmat H, Karbasi-Afshar R, Agah S, Taheri S. Helicobacter pylori Infection in the general population: A Middle Eastern perspective. Caspian J Intern Med. 2013;4(4):745-753.

26. Armstrong D. Helicobacter pylori infection and dyspepsia. Scand J Gastroenterol Suppl. 1996;215:38-47.

27. Talley NJ, Ford AC. Functional Dyspepsia. N Engl J Med. 2015;373(19):1853-1863.

28. de Jong JJ, Lantinga MA, Drenth JP. Prevention of overuse: A view on upper gastrointestinal endoscopy. World J Gastroenterol. 2019;25(2):178-189.

29. Cardin F, Zorzi M, Bovo E, Guerra C, Bandini F, Polito D, Bano F, et al. Effect of implementation of a dyspepsia and Helicobacter pylori eradication guideline in primary care. Digestion. 2005;72(1):1-7. 